\title{
PERFIL NUTRICIONAL DAS FAMÍLIAS DA VILA TECNOLÓGICA NO MUNICÍPIO DE RIBEIRÃO PRETO
}

\author{
Maria das Graças Canvalho Ferriani \\ Rosane Pilot Pessa Ribeiro ${ }^{2}$ \\ Moacyr Lobo da Costa Junior ${ }^{3}$ \\ Graziela Vieira Bassan dos Santos ${ }^{4}$ \\ Marta Neves Campanelli Marçal Vieira ${ }^{4}$ \\ Juliana da Silva Camargo 5 \\ Daniela Vieira
}

\section{INTRODUÇÃO}

O problema de carência nutricional no Brasil atinge 32 milhões de brasileiros, número equivalente a $22 \%$ da população do país ou 9 milhões de famílias. Tais dados não se explicam pela disponibilidade interna de alimentos ou fatores econômicos isoladamente, mas tem raízes multissetoriais. O poder aquisitivo e certos aspectos de produção, colheita, armazenamento e transporte de alimentos, além certamente de hábitos É importante ressaltar que os resultados da pesquisa indicam que em cada 5 famílias, 4 apresentam problemas nutricionais. alimentares inadequados, são fatores envolvidos na alta prevalência de carência nutricional.

Em contraste com esses dados de desnutrição por várias formas, a obesidade atinge mais de $5 \%$ das crianças menores de 5 anos, mesmo em famílias de baixa renda nas regiões Sul e Sudeste. A partir dos 18 anos de idade, o sobrepeso e a obesidade tornam-se cada vez mais freqüentes: cerca de $32 \%$ dos brasileiros adultos apresentam algum grau de excesso de peso (Oliveira,1996)

Diante desses dados, tudo indica que a situação nutricional da população vem sofrendo mudanças, por um lado, com evidente diminuição das taxas de desnutrição, e por outro lado, com o aumento da obesidade associada ao aumento da morbi-mortalidade pelas doenças crônico-degenerativas (hipertensão arterial, dislipidemia, neoplasias malignas, diabete mélitus), ligadas direta ou indiretamente a exposição a determinados nutrientes.

Considerando todos estes aspectos, justifica-se o nosso interesse em estudar o estado nutricional das famílias de um bairro da periferia de Ribeirão Preto, que fazem parte do "Projeto de Vigilância em Saúde das Famílias da Vila Tecnológica" coordenado pela Escola de Enfermagem de Ribeirão PretoUSP e Secretaria Municipal da Saúde de Ribeirão Preto, com vistas a subsidiar a Implantação de um Sistema de Vigilância Alimentar Nutricional.

Sendo assim, o objetivo do presente estudo foi avaliar o estado nutricional das famílias residen-

1 Prof ${ }^{a}$ Dra. Titular do Depto. de Enfermagem Materno-Infantil e Saúde Pública da Escola de Enfermagem de Ribeirão Preto da Universidade de São Paulo.

2 Profa Dra. do Depto. de Enfermagem Materno-Infantil e Saúde Pública da Escola de Enfermagem de Ribeirão Preto da Universidade de São Paulo.

${ }^{3}$ Prof. Dr. do Depto. de Enfermagem Materno-Infantil e Saúde Pública da Escola de Enfermagem de Ribeirão Preto da Universidade de São Paulo.

${ }^{4}$ Nutricionista Doutoranda da Escola de Enfermagem de Ribeirão Preto da Universidade de São Paulo.

${ }^{5}$ Alunas da Escola de Enfermagem de Ribeirão Preto da Universidade de São Paulo. 
FERRIANI, Maria das Graças Carvalho et al.

tes na Vila Tecnológica do município de Ribeirão Preto.

\section{METODOLOGIA}

A pesquisa foi realizada no bairro "Vila Tecnológica", e tem como sede para o desenvolvimento do projeto de "Vigilância em Saúde das Famílias da Vila Tecnológica", a Casa da Saúde, implantada em 1997, que é uma Unidade de Atenção Primária à Saúde (APS) ligada à Secretaria Municipal da Saúde, situada ao oeste da cidade. Conta com uma enfermeira, uma auxiliar de enfermagem e uma auxiliar de serviço. As atividades desenvolvidas na Casa da Saúde voltam-se para a prevenção de doenças e promoção da saúde.

A Casa da Saúde situa-se estrategicamente dentro de um espaço denominado Rua das Tecnologias ${ }^{6}$, espaço este sob a coordenação da Secretaria da Cidadania e Desenvolvimento Social do município, onde são desenvolvidas ações de caráter educativo e de promoção social, principalmente com crianças e adolescentes de toda a região.

A pesquisa foi realizada com 106 famílias cadastradas na Casa de Saúde, que residem no bairro da Vila Tecnológica, correspondendo a um contingente de 471 pessoas. As famílias receberam inicialmente orientação sobre o estudo e foram agendadas para aferição do peso e da estatura, através de aerograma, na Casa da Saúde.

Considerando que um número expressivo de famílias não compareceu ao local, foi efetuada a visita domiciliar para coleta de dados que se realizou no período de agosto de 1998 a janeiro de 1999.

A medida de peso foi obtida utilizando balança antropométrica (marca FILIZOLA) portátil, com capacidade de $150 \mathrm{~kg}$ e escala com divisões em quilogramas e 100 gramas. A altura foi obtida com uma régua de madeira graduada em centímetros. A coleta de dados foi realizada por duas alunas de graduação do curso de enfermagem sendo os dados anotados em impresso próprio. Utilizamos um veículo oficial com motorista da Escola de Enfermagem de Ribeirão Preto-USP para transporte das mesmas até o local de estudo.

Após o levantamento dos dados antropométricos foi efetuada a classificação do estado nutricional por grupos etários. O estudo fez uso das variáveis peso, altura, sexo e idade, utilizando-se para a faixa etária até 10 anos, os três indicadores: peso por idade (P/I), isto é, a relação entre o peso de referência por altura e altura por idade (A/l), relação entre a altura observada e a altura de referência por idade. Os dados peso, altura, sexo, idade foram digitados no software EPI INFO, no qual calculou-se os escores Z, sendo posteriormente transferidos para o Diagnutri (Goulart, Basques, 1991) que calculou a distribuição de freqüência dos escores $Z$ observados na amostra em estudo. Para a faixa etária acima de 10 anos até 18 anos foi utilizado o Indice de Massa Corporal (IMC) para adolescentes de acordo com dados obtidos da "National Health and Nutrition Examination Survey" ( WHO, 1995).

Para os adultos foi utilizado o IMC em relação à idade expresso pela obtenção do cálculo do peso dividido pela altura ao quadrado, comparado com a classificação seguida por Bray(1996).

\section{RESULTADOS}

Os dados coletados podem ser apresentados e analisados sob dois prismas distintos: a família como unidade de análise ou considerando-se o indivíduo.

${ }^{6}$ A Rua das Tecnologias foi criada em 1995 dentro de um projeto habitacional da COHAB - Companhia Habitacional do município de Ribeirão Preto, visando a experimentação de formas alternativas de construção habitacional de baixo custo. Existem cerca de 12 construções, sendo que inicialmente a Rua serviu de área de exposição e, posteriormente, foi ocupada dentro de uma proposta da Secretaria de Bem estar Social do município. Assim, na Rua das tecnologias, encontram-se casas de um mesmo padrão de construção mas que apresentam distintos materiais e tecnologias de construção (madeira, placas de concreto, gesso, isopor, etc). Atualmente, esta rua apresenta uma extensa área de lazer que é ocupada por toda a população da região e as casa construídas, são utilizadas por entidades governamentais e não governamentais numa perspectiva de assistência social. 
Com relação às famílias, podemos visualizar na Quadro1 que, das 106 famílias existentes, 101 (99\%) foram contatadas, sendo que em 08 delas não foi possível fazer a avaliação nutricional em nenhum de seus membros.

Outro ponto a destacar é que, nas 93 famílias em que foi realizada a avaliação nutricional, observam-se- em 08 famílias ninguém apresentou problemas relacionados à nutrição, tendo sido todas as pessoas examinadas e em 10 famílias, não se constatou nenhum problema nutricional, porém, nem todos os familiares foram examinados. Portanto, nas 75 famílias restantes, o que corresponde a $71 \%$ das famílias existentes na Vila tecnológica, ou $81 \%$ das famílias onde foram examinadas pelo menos uma pessoa, em todas elas, observou-se pelo menos um membro com problema nutricional.

QUADRO 1: Distribuição das famílias segundo resultado da avaliação do estado nutricional em seus elementos.

\begin{tabular}{|l|c|}
\hline \multicolumn{1}{|c|}{ Avaliação } & $\begin{array}{c}\text { Número de } \\
\text { Famílias }\end{array}$ \\
\hline Não encontrada & 5 \\
Encontrada e não avaliada & 8 \\
Avaliada - nem todos os familiares & 10 \\
Avaliada - todos normais & 8 \\
Avaliada - pelo menos 1 desnutrido ninguém & 14 \\
obeso & 39 \\
Avaliada - pelo menos 1 obeso ninguém & \\
desnutrido & 22 \\
Avaliada - pelo menos 1 desnutrido e um obeso. & 106 \\
\hline Total de famílias & \\
\hline
\end{tabular}

No que concerne ao número de pessoas, nas 101 famílias que participaram do estudo há um contingente de 471 pessoas, sendo que dessas, 98 não foram avaliadas quanto a o seu estado nutricional, em decorrência de vários fatores.

Em relação às 373 pessoas que foram avaliadas, constatou-se que $217(58 \%)$ destas apresentaram valores normais de peso e altura. Entretanto, 156 (42\%) pessoas apresentaram algum agravo nutricional, sendo

54 (15\%) pessoas desnutridas e 102 (27\%) obesas. Do contingente de indivíduos desnutridos, 31 deles $(42,6 \%)$ são crianças de 0 a 9 anos e dos 102 obesos, 23 deles $(20,9 \%)$ são adolescentes entre 10 e 19 anos e $64(30,4 \%)$ são adultos, prevalecendo os do sexo feminino $(61,7 \%)$.

É importante ressaltar que os resultados da pesquisa indicam que em cada 05 famílias, 04 apresentam problemas nutricionais.

Comparando-se os dados da presente pesquisa com a literatura, observa-se que estes não são tão diferentes, entretanto, há necessidade de se conhecer o consumo alimentar das famílias pesquisadas, a forma de aquisição de alimentos e dados de morbidade por faixa etária.

Em Ribeirão Preto, uma pesquisa realizada por Santos citado por Pereira (1995), em quatro bairros da cidade, verificou que a prevalência de adultos com peso até $30 \%$ acima do ideal foi de $48 \%$ e, o de mais de $30 \%$ acima do ideal foi de $17,7 \%$. Outro estudo, realizado em Ribeirão Preto por Santos, (1997) com pré-escolares, esta encontrou 17,4\% de crianças com desnutrição moderada ou grave. A prevalência de obesidade foi de 10,2\%, ou seja, crianças que apresentaram relação de peso para altura acima de 2 escores $Z$.

A obesidade e a desnutrição têm sido vistas como problemas mundiais de saúde pública e que também necessitam de atenção por parte dos governos e dos profissionais de saúde.

O estado de saúde e nutrição depende da forma como as crianças e seus familiares estão inseridos na sociedade e a qualidade do ambiente vivenciado por elas. Estes aspectos deverão ser analisados ao se discutir desnutrição e sobrepeso ou obesidade.

Outro aspecto relevante é o acesso à educação que contribui sobre maneira para o estado de saúde e nutrição dos indivíduos, em conjunto com outros fatores do meio social, permitindo a melhoria 
das condições de vida de trabalho, de renda e consequentemente, possibilitando o acesso à alimentação.

Monteiro et al. (1995), refletem sobre a transição nutricional no Brasil e concluem que esta determinará a definição de prioridades e estratégias de ação de Saúde Pública, fazendo com que o país incorpore de vez a prevenção e o controle das doenças crônicas degenerativas, elaborando ações de educação em alimentação e nutrição que alcancem eficazmente todos os estratos econômicos.

\section{CONSIDERAÇÕES FINAIS}

Os resultados da pesquisa possibilitaram conhecer o estado nutricional dessas famílias, embora, uma análise mais aprofundada seja necessária para conclusão deste estudo. Entretanto, constatou-se que estas famílias apresentam também uma polarização em seu quadro nutricional convivendo com duas realidades: desnutrição infantil e obesidade na fase adulta. Desta forma são necessárias algumas medidas para que se possa reverter tal quadro.

Considerando a nossa proposta de "Implantação de um Sistema de Vigilância Nutricional Familiar, pretende-se:

- realização de visita domiciliar por amostragem para levantar dados sobre consumo alimentar das famílias e formas de aquisição de alimentos (compra, doação, produção doméstica, dentre outros);

- análise dos resultados da avaliação do estado nutricional e do inquérito alimentar associando aos dados de morbidade já levantados em outra instância com vistas a subsidiar o plano de orientação alimentar e nutricional às famílias.

- elaborar proposta de um sistema de vigilância alimentar e nutricional que envolva:

- programas de educação alimentar (casa da saúde, escolas, creches, UBS, dentre outros);

- suplementação alimentar para grupo de risco (lactantes, pré-escolares, adolescentes, gestantes, idosos e indivíduos enfermos);

- programas de abastecimento alimentar e de vigilância sanitária de alimentos.

- programa de acompanhamento do estado nutricional da população.

\section{REFERÊNCIAS BIBLIOGRÁFICAS}

BRAY, G.A. Obesity-Endocrinology and Metabolism. Clinics of North America. W.B. Saunders, v. 25, n. 4, December, 1996.

GOULART, E.M.A.; BASQUES, S.A. DIAGNUTRI, versão 1.0: diagnóstico da desnutrição infantil. Belo Horizonte/ MG/Departamento de Pediatria da Faculdade de Medicina da UFMG, 1991.

MONTEIRO, C.A.; MONDINI, L; SOUZA, A.L.M.; POPKIN, B.M. Da desnutrição para a obesidade: a transição nutricional no Brasil. In: MONTEIRO, C.A. (Org.) Velhos e novos males da saúde no Brasil. São Paulo: HUCITEC, 1995. p.248-54.

OLIVEIRA, J.E.D. Uma análise da nutrição no Brasil: In: Crescimento Saudável. Informativo Quadrimestral para Médicos e Nutricionistas, n.1., jun./set. 1996.

PEREIRA, S. Classe alta tem maior tendência à obesidade. Folha Nordeste. Folha de São Paulo, 30 de julho de 1995. Folha Ribeirão.

SANTOS, G.B.S. Desnutrição e obesidade em pré-escolares da rede municipal de Ensino de Ribeirão PretoSP. Ribeirão Preto, 1997. 151 p. Dissertação (Mestrado) - Escola de Enfermagem de Ribeirão Preto, Universidade de São Paulo.

WHO. Obesity. preventing and managing the global epidemic. Report of a WHO Consultation on Obesity. Geneva: World Health Organization, 1998. 